\title{
Vorteil durch Eigenständigkeit? Analyse der Publikationsleistung der universitären Plastischen Chirurgie in verschiedenen Organisationsstrukturen
}

\author{
Independence in Plastic Surgery - Benefit or Barrier? Analysis of the Publication \\ Performance in Academic Plastic Surgery Depending on Varying Organisational Structures
}

Autoren

Institut

\section{D. Schubert*, S. Leitsch*, F. Haertnagl, E. M. Haas, R. E. Giunta}

Handchirurgie, Plastische Chirurgie und Ästhetische Chirurgie, Campus Großhadern und Campus Innenstadt, Klinikum der Ludwig-Maximilians Universität München (LMU), München
Schlüsselwörter

- Akademische Plastische Chirurgie

- Forschung

- Eigenständigkeit

- Pubmed-Analyse

- Organisationsstruktur

Key words

- academic plastic surgery

- research outcome

- independence

- pubmed analysis

- organisational structure

eingereicht $\quad$ 12.6.2015

akzeptiert $\quad 6.7 .2015$

Bibliografie

DOI http://dx.doi.org/

10.1055/s-0035-1555954

Handchir Mikrochir Plast Chir

2015; 47: 213-221

(c) Georg Thieme Verlag KG

Stuttgart · New York

ISSN 0722-1819

Korrespondenzadresse

Dr. med. Cornelius Dieter

\section{Schubert, MD}

Handchirurgie, Plastische Chirurgie und Ästhetische Chirurgie Klinikum der Ludwig-Maximilians Universität München (LMU)

Pettenkoferstraße 8a

80336 München

cornelius.schubert@med.

uni-muenchen.de

\section{Zusammenfassung}

$\nabla$

Einleitung: Trotz der Anerkennung als eigenständiges Fachgebiet ist die Plastische Chirurgie in der deutschen Universitätsmedizin noch unzureichend im Sinne unabhängiger Kliniken mit entsprechendem Forschungsfokus verankert. Ziel dieser Arbeit war es, die Publikationsleistung der deutschen akademischen Plastischen Chirurgie zu untersuchen und einen Vergleich zwischen eigenständigen Kliniken und untergeordneten Organisationsstrukturen zu ziehen. Material und Methodik: Mittels WebseitenAnalyse wurden Organisationsstrukturen und personelle Ausstattung an Universitätsklinika errmittelt. Via Pubmed-Analyse wurde die Publikationsleistung (Publikationsanzahl, kumulativer Impactfaktor, Impactfaktor/Publikation, Publikationen/Arzt, Publikationen/Klinik) im Zeitraum von 2009 bis 2013 eruiert. Die Verteilung von kumulativem Impactfaktor und Publikationsanzahl auf die verschiedenen Fachzeitschriften sowie die Entwicklung des Impactfaktors wurde in einer Journal-Analyse untersucht. Ergebnisse: An den 35 Universitätsklinika existierten 12 eigenständige Kliniken und 8 untergeordnete Organisationsstrukturen. An 15 Universitätsklinika gab es keine plastisch-chirurgischen Organisationsstrukturen. Der Personalschlüssel unterschied sich deutlich zwischen Kliniken (3,6 OberärztInnen/Einheit) und untergeordneten Organisationsstrukturen (1,1 OberärztInnen/Einheit). Der Großteil der Publikationen $(89,0 \%)$ und kumulativen Impactfaktorpunkte $(91,2 \%)$ sowie die meisten Publikationen/ Arzt (54 Publikationen/Jahr) und pro Klinik (61 Publikationen/Jahr) waren an den eigenständigen Kliniken zu finden. Nur hier gab es Top-Publikationen mit einem Impactfaktor $>5$. Es zeichnete sich ein allgemeiner Negativtrend bzgl. Publikationszahl (-13,4\%) und kumulativen

${ }^{*}$ Geteilte Erstautorenschaft.

\section{Abstract \\ $\nabla$}

Introduction: Despite its recognition as an independent specialty, at German university hospitals the field of plastic surgery is still underrepresented in terms of independent departments with a dedicated research focus. The aim of this study was to analyse the publication performance within the German academic plastic surgery environment and to compare independent departments and dependent, subordinate organisational structures regarding their publication performance.

Material and Methods: Organisational structures and number of attending doctors in German university hospitals were examined via a website analysis. A pubmed analysis was applied to assess the publication performance (number of publications, cumulative impact factor, impact factor/publication, number of publications/MD, number of publications/unit) between 2009 and 2013. In a journal analysis the distribution of the cumulative impact factor and number of publications in different journals as well as the development of the impact factor in the top journals were analysed.

Results: Out of all 35 university hospitals there exist 12 independent departments for plastic surgery and 8 subordinate organisational structures. In 15 university hospitals there were no designated plastic surgery units. The number of attending doctors differed considerably between independent departments (3.6 attending doctors/unit) and subordinate organisational structures (1.1 attending doctors/unit). The majority of publications (89.0\%) and of the cumulative impact factor $(91.2 \%)$ as well as most of the publications/MD (54 publications/year) and publications/ unit (61 publications/year) were created within the independent departments. Only in departments top publications with an impact factor $>5$ were published. In general a negative trend 
Impactfaktorpunkten (-28,9\%) ab. 58,4\% aller Publikationen verteilten sich auf die Top-10 Zeitschriften, wovon ein Großteil (60\% der Publikationen, 79,7\% des kumulativen Impactfaktors) auf englischsprachige Fachzeitschriften entfiel. Der Impactfaktor der Top-10 Fachzeitschriften entwickelte sich im Untersuchungszeitraum positiv (+13,5\%).

Schlussfolgerung: Eigenständige Kliniken für Plastische Chirurgie sind im Gegensatz zu untergeordneten Organisationsstrukturen die zentralen Leistungsträger innerhalb der akademischen Plastischen Chirurgie, welche im Allgemeinen bzgl. der Publikationsleistung rückläufige Tendenzen zeigt. Die Art der Organisationsstruktur hat somit entscheidenden Einfluss auf die wissenschaftliche Leistungsfähigkeit.

\section{Einleitung}

$\nabla$

Die Plastische Chirurgie hat sich als junges chirurgisches Fachgebiet in den vergangenen Jahrzehnten zunehmend etabliert. Ihre zentrale Bedeutung innerhalb der Chirurgie ist heute unumstritten und aus dem klinischen sowie wissenschaftlichen Alltag nicht mehr weg zu denken. Angefangen in den 50er Jahren mit der Gründung der ersten Abteilung für Plastische Chirurgie unter der Leitung von Frau Ursula Schmidt-Tintemann am Klinikum rechts der Isar der Technischen Universität München ist die Plastische Chirurgie inzwischen an zahlreichen Universitätsklinika etabliert [1]. Parallel zur Zunahme der Kliniken für Plastische Chirurgie ist auch eine entsprechend exponentiell steigende Zunahme an Fachärzten für Plastische und Ästhetische Chirurgie zu verzeichnen [1]. Trotz der ständig zunehmenden Bedeutung der Plastischen Chirurgie innerhalb der Patientenversorgung, trotz des beträchtlichen wissenschaftlichen Outputs und eines geschärften und klar definierten Aufgabenprofils sind an deutschen Universitätsklinika eigenständige Kliniken bzw. Lehrstühle für Plastische Chirurgie immer noch eher die Ausnahme als die Regel. Es wäre nicht überraschend, wenn sich diese aktuell noch nicht ausreichende Verankerung der Plastischen Chirurgie an den deutschen Universitätsklinika auch in einer entsprechend ungleichmäßig verteilten Publikationsleistung widerspiegeln würde. Viele Faktoren beeinflussen die Publikationsleistung. Angefangen von der Qualifikation der wissenschaftlichen Mitarbeiter über die Zeitressourcen, die für wissenschaftliche Arbeit zur Verfügung stehen, bis hin zur LaborINFRAstruktur innerhalb der Klinik. Eine zunehmende Autonomie im Sinne von Lehrstühlen mit eigenständigem Management, erhöhten Mitarbeiterzahlen, größeren finanziellen und räumlichen Mitteln, Leistungs-orientierten Mitteln (LOM) sowie der Möglichkeit des Aufbaus einer strukturierten Forschungsinfrastruktur könnten durchaus positive Rahmenbedingungen für eine leistungsfähige und international konkurrenzfähige Forschung bieten. Auch andere „kleinere“ chirurgische Fachgebiete wie z.B. die Gefäßchirurgie berichteten bereits von ersten vorteilhaften Auswirkungen einer zunehmenden Autonomie auf die Forschungsleistung $[2,3]$. Aber nicht nur in Deutschland sondern auch international z.B. in den USA sind der Vorteil zunehmender Eigenständigkeit sowie die mangelnde Emanzipation der akademischen Plastischen Chirurgie im Sinne autonomer Kliniken ein kontrovers diskutiertes Thema [4]. So existierten im Jahre 2007 an allen US Medical Schools lediglich 8 eigenständige Departments of Plastic Surgery $[4,5]$.

Über den momentanen Stand der Publikationsleistung der universitären Plastischen Chirurgie insbesondere in Hinblick auf regarding the number of publications $(-13.4 \%)$ and cumulative impact factor $(-28.9 \%)$ was observed. $58.4 \%$ of all publications were distributed over the top 10 journals. Within the latter the majority of articles were published in English journals (60\% of publications, $79.9 \%$ of the cumulative impact factor). The average impact factor of the top 10 journals increased by $13.5 \%$ from 2009 to 2013.

Conclusion: In contrast to subordinate and dependent organisational structures, independent departments of plastic surgery are the key performers within German academic plastic surgery which, however, suffers from a general declining publication performance. Hence, the type of organisational structure has a crucial influence on the research performance.

verschiedene Organisationsstrukturen existieren wenige aktuelle Studien [2].

Ziel dieser Arbeit war es, im Rahmen einer Webseiten-, Pubmedsowie Journal-Recherche die aktuelle Publikationsleistung der akademischen Plastischen Chirurgie in Deutschland zu untersuchen und zugleich einen Vergleich zwischen eigenständigen Kliniken und untergeordneten Organisationsstrukturen zu ziehen.

\section{Material und Methode}

$\nabla$

\section{Webseiten-Analyse}

Ob an einem Universitätsklinikum überhaupt eine Plastische Chirurgie vorhanden ist bzw. welche Organisationsstrukturen dort vorherrschen ( $\bullet$ Tab. 1) wurde anhand einer Analyse der jeweiligen Klinik-Webseiten untersucht. Die Anzahl der jeweils beschäftigten Chef- und Oberärzte wurde zum Stand 31.12.2013 festgehalten. Zu den offiziell im Verband der Universitätsklinika Deutschlands e.V. (VUD) gelisteten 33 ordentlichen Mitgliedern wurden zusätzlich die Universität Oldenburg als assoziiertes Mitglied der VUD sowie die private Universität Witten/Herdecke hinzu gerechnet [6]. Der in Nürnberg errichtete Standort der österreichischen Paracelsus Medizinische Privatuniversität wurde aufgrund seines Sonderstatus einer österreichischen Privatuniversität innerhalb Deutschlands aus der Studie ausgeschlossen.

\section{Pubmed-Analyse}

Die Publikationsleistung der universitären Kliniken wurde anhand einer Pubmed-Recherche der Jahre 2009-2013 erfasst. Aufgenommen wurden alle Pubmed-gelisteten Artikel, welche von Chef- oder Oberärzten verfasst wurden, die im Rahmen der Webseiten-Analyse (s.o.) an einer deutschen universitären Plastischen Chirurgie tätig waren. Gezählt wurden nur Erst- oder Letztautorenschaften. Waren jeweils Erst- und Letztautor universitäre Plastische Chirurgen, so wurde der Artikel entsprechend doppelt - einmal für den Erstautor und ein zweites Mal für den Letztautor - gezählt. Koautorenschaften wurden ausgeschlossen. Waren Ärzte vormals an einem anderen Universitätsklinikum tätig, wurden alle Publikationen aus dem Untersuchungszeitraum der Klinik zugesprochen, an der der Arzt zum 31.12.2013 laut Klinik-Webseiten gelistet war. Die Analyse beschränkt sich auf Universitätsklinika. Lehrkrankenhäuser wurden nicht gewertet. Stand der Pubmed-Analyse ist der 10.06.2014. Der Journal Citation Index (JCI) des Jahres 2012 wurde als Grundlage für die anschließende Impactfaktor-Auswertung herangezogen. 


\begin{tabular}{|c|c|c|c|}
\hline $\begin{array}{l}\text { Organisations- } \\
\text { struktur }\end{array}$ & Führungsstruktur & Verantwortlichkeiten & $\begin{array}{l}\text { Definition im } \\
\text { Artikel }\end{array}$ \\
\hline Klinik & $\begin{array}{l}\text { - Lehrstuhl } \\
\text { - Ordinariat } \\
\text { - W3 Professur }\end{array}$ & $\begin{array}{l}\text { - Eigenes Budget } \\
\text { - Eigene Forschungs-, Personal-, Raum-, } \\
\text { OP-, Ambulanzressourcen } \\
\text { - LOM Mittel } \\
\text { - Unabhängige Strategieplanung }\end{array}$ & $\begin{array}{l}\text { Eigenständige } \\
\text { Kliniken }\end{array}$ \\
\hline Abteilung & $\begin{array}{l}\text { - W3 oder W2 Professur } \\
\text { - Extraordinariat } \\
\text { - Abteilungsdirektor }\end{array}$ & $\begin{array}{l}\text { - Eigenes Budget } \\
\text { - Eigene Forschungs-, Personal-, Raum-, } \\
\text { OP-, Ambulanzressourcen } \\
\text { - LOM Mittel } \\
\text { - Abhängige Strategieplanung }\end{array}$ & \multirow[t]{2}{*}{$\begin{array}{l}\text { Untergeordnete } \\
\text { Organisations- } \\
\text { strukturen }\end{array}$} \\
\hline Sektion & $\begin{array}{l}\text { - W2 Professur } \\
\text { - Chefarzt oder Oberarzt }\end{array}$ & $\begin{array}{l}\text { Alle Ressourcen } \\
\text { durch übergeordnetes } \\
\text { Fachgebiet kontrolliert } \\
\text { und disponiert }\end{array}$ & \\
\hline Einzelner Oberarzt & Oberarzt & Keine Ressourcen & \multirow{2}{*}{$\begin{array}{l}\text { Keine } \\
\text { ausgewiesene } \\
\text { Organisations- } \\
\text { struktur }\end{array}$} \\
\hline $\begin{array}{l}\text { Keine } \\
\text { Plastische Chirurgie }\end{array}$ & $\begin{array}{l}\text { Teilaspekte des } \\
\text { Fachgebietes von anderen } \\
\text { Fachgebieten abgebildet }\end{array}$ & Keine Ressourcen & \\
\hline
\end{tabular}

Tab. 1 Möglichkeiten der Repräsentation des Fachgebietes Plastische und Ästhetische Chirurgie an deutschen Universitätsklinika.
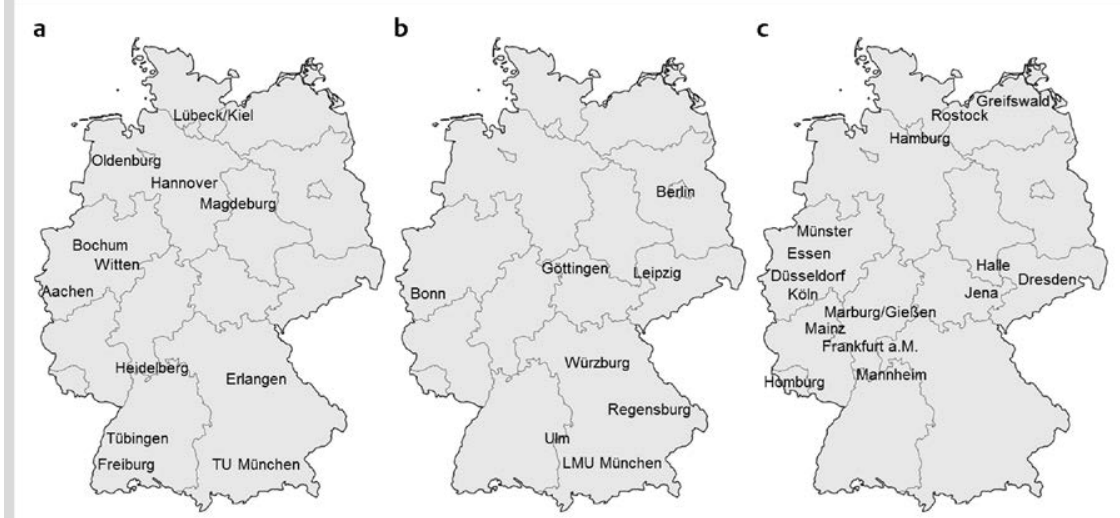

Abb. 1 Organisationsstrukturen der Plastischen Chirurgie an deutschen Universitätsklinika a Eigenständige Kliniken für Plastische Chirurgie b Untergeordnete Organisationsstrukturen c Universitätsklinika ohne ausgewiesene plastisch-chirurgische Organisationsstrukturen. Stand: 31.12 .2013

\section{Limitationen}

Einschränkend muss erwähnt werden, dass die im Ausland erbrachten Publikationsleistungen einzelner Ärzte nicht gewertet wurden. Ein individuelles Nachvollziehen einzelner Lebensläufe war teils nicht möglich. Zudem wurden Ärzte, unabhängig von teils hohen Publikationsoutcomes, nicht in die Analyse aufgenommen wenn diese zum Stichtag 31.12.2013 bereits die Universitätsklinik verlassen hatten. Diese Analyse bildet daher jeweils einen groben Trend ab und stellt keinen Anspruch auf absolute Vollständigkeit.

\section{Journal-Analyse}

Zur Untersuchung der Impactfaktor-Entwicklung der meistveröffentlichen Fachzeitschriften im Verlauf von 5 Jahren wurden der Journal Citation Index (JCI) der Jahre 2009 und 2013 verglichen.

\section{Statistik}

Die Unterschiede zwischen den einzelnen Gruppen wurden mittels eines einseitigen T-Tests und unter Annahme gleicher Varianz auf ihre Signifikanz hin analysiert. Eine Irrtumswahrscheinlichkeit kleiner $5 \%(\mathrm{p}<0,05)$ galt als signifikant, ein Wert kleiner $1 \%(\mathrm{p}<0,01)$ als hochsignifikant. Alle Berechnungen wurden mit der Software Microsoft Office Excel 2013 erstellt (Microsoft Corporation, Redmond, WA, USA)

\section{Ergebnisse}

\section{Webseiten-Analyse}

Gemäß der Webseiten der Universitätsklinika, waren zum 31.12.2013 an lediglich 12 der 35 Universitätsklinika Lehrstühle für Plastische Chirurgie etabliert ( $\bullet$ Abb. 1a). An 23 Universitätsklinika gab es keine selbständige Klinik bzw. Lehrstuhl für Plastische Chirurgie ( $\bullet$ Abb. 1a+b). An 15 Universitätsklinika gab es auf der Webseite überhaupt keine ausgewiesene plastisch-chirurgische Organisationsstrukturen ( $\bullet$ Abb. 1c). An 8 Universitätsklinika war eine universitäre Plastische Chirurgie im Rahmen untergeordneter Organisationsstrukturen aufzufinden. Diese variierten jeweils deutlich von formal untergeordneten Sektionen innerhalb übergeordneter Kliniken über privat geführte Einheiten oder im Rahmen von Klinik-übergreifenden Zentren für Plastische Chirurgie, sodass innerhalb der untergeordneten Organisationsstrukturen auf eine weitere Aufteilung verzichtet wurde. Auch der Personalschlüssel unterschied sich deutlich mit 3,6 OberärztenInnen pro Einheit (Range: 2-7 OÄ/Einheit) an eigenständigen Kliniken. Im Gegensatz dazu waren an den Kliniken, welche überhaupt untergeordnete Organisationsstrukturen anboten, nur 1,1 OberärztInnen pro Einheit (Range: 0-4 OÄ/Einheit) tätig. Teilweise war hier nur ein einzelner Chefarzt bzw. Sektionsleiter ohne weitere Oberärzte gelistet. Insgesamt waren 
zum Stichtag 55 Ober- bzw. Chefärzte an selbstständigen Kliniken für Plastische Chirurgie und 18 Ober- bzw. Chefärzte an Organisationsstrukturen gelistet, die anderen Fachgebieten untergeordnet waren. Dies entspricht einem Verhältnis von ca. 3:1.

\section{Pubmed-Analyse}

Im Rahmen der Pubmed-Recherche stellte sich heraus, dass der Großteil der Publikationen von den selbstständigen Kliniken für Plastische Chirurgie veröffentlicht wurde. So wurden von insgesamt 946 Publikationen im 5-Jahres-Zeitraum zwischen 2009 und 2013842 Publikationen und somit 89,0\% der Gesamtpublikationen an den 12 Kliniken für Plastische Chirurgie erbracht ( $\bullet$ Abb. 2). Im selben Zeitraum wurden von den untergeordneten Organisationsstrukturen lediglich 104 Publikationen veröffentlicht. Im Vergleich zu den untergeordneten Organisationsstrukturen veröffentlichten die autonomen Kliniken damit über 8-mal so viele Publikationen. Im 5-Jahresvergleich sank die Gesamtzahl der Publikationen von ursprünglich 194 Publikationen/Jahr (2009) um 13,4\% auf 168 Publikationen/Jahr (2013). Während über den Zeitraum von 5 Jahren die eigenständigen Kliniken jedoch von 181 Publikationen/Jahr (2009) um 21,5\% auf 142 Publikationen/Jahr (2013) sanken, stieg die Publikationsleistung der untergeordneten Organisationsstrukturen um $100,0 \%$ von 13 Publikationen (2009) auf 26 Publikationen (2013). Bei Betrachtung des entsprechenden kumulativen Impactfaktors zeichnet sich ein noch eindrücklicheres Bild ab ( A Abb. 3). Auch hier bestätigt sich die hohe Publikationsleistung der eigenständigen Kliniken im Vergleich zu den untergeordneten Organisationsstrukturen. Im Gesamtzeitraum zwischen 2009-2013 wurde insgesamt ein kumulativer Impactfaktor von 1709,34 Punkten erbracht. Davon entfiel auf die selbstständigen Kliniken ein kumulativer Impactfaktor von 1559,77 Punkten, was einen Anteil von 91,2\% der Gesamt-Impactfaktorpunkte darstellt. Im Vergleich ist der Anteil der untergeordneten Organisationsstrukturen marginal mit 149,57 kumulativen Impactfaktorpunkten, was einen Anteil von 8,8\% der Gesamtleistung wiederspiegelt. Der gesamte kumulative Impactfaktor sank im 5-Jahresvergleich um 28,9\% von 396,13 Punkte (2009) auf 281,63 Punkte (2013). Der kumulative Impactfaktor sank bei den eigenständigen Kliniken im 5-Jahresvergleich von 371,15 Punkten (2009) um 31,0\% auf 255,91 Punkte (2013). Im Vergleich dazu verbesserte sich der kumulative Impactfaktor der
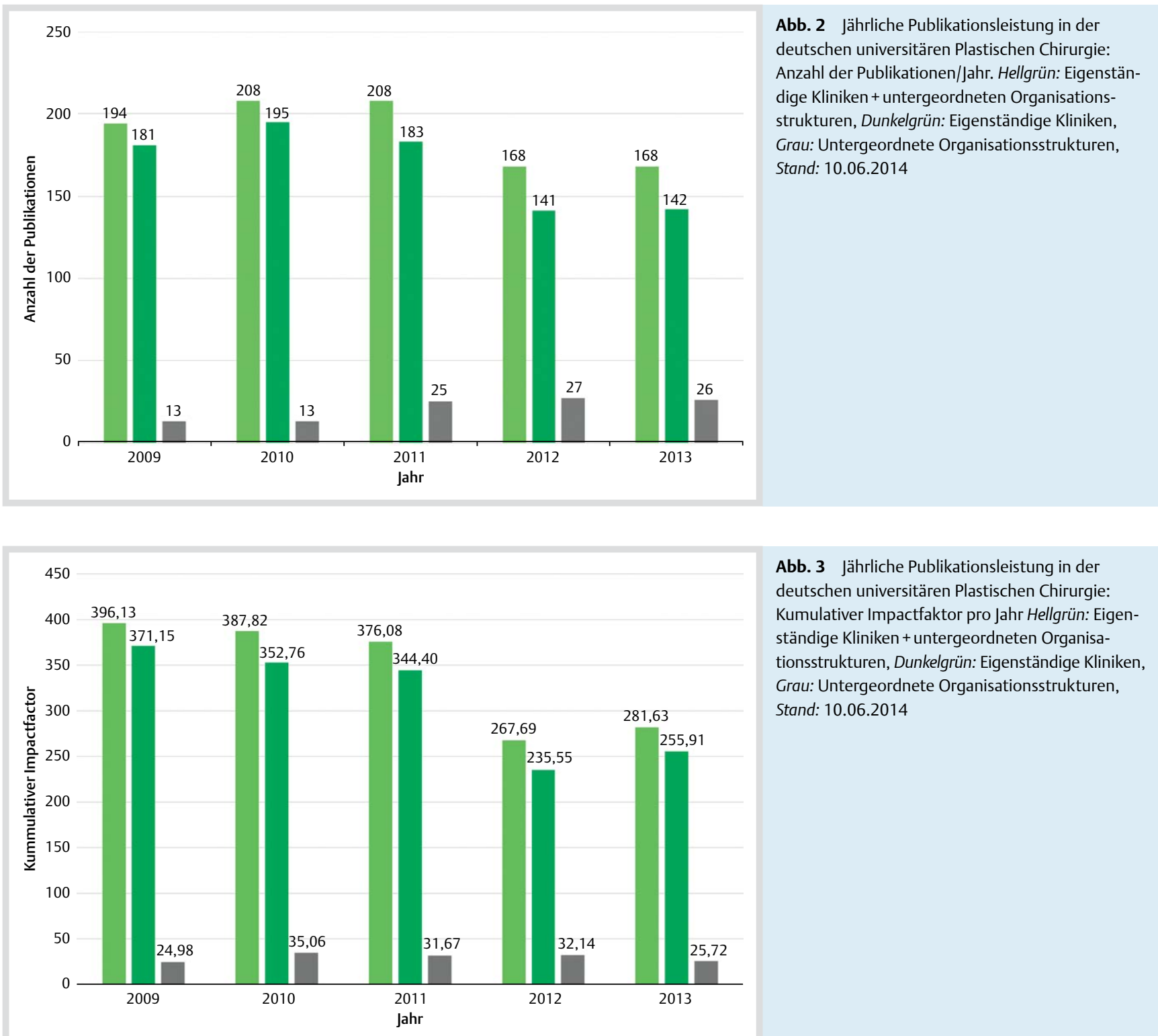

Abb. 3 Jährliche Publikationsleistung in der deutschen universitären Plastischen Chirurgie: Kumulativer Impactfaktor pro Jahr Hellgrün: Eigenständige Kliniken + untergeordneten Organisationsstrukturen, Dunkelgrün: Eigenständige Kliniken, Grau: Untergeordnete Organisationsstrukturen, Stand: 10.06 .2014 
untergeordneten Organisationsstrukturen von ursprünglich 24,98 Punkten (2009) um 3,0\% (2013) auf 25,72 Punkte nur marginal.

Die Publikationsanzahl und der kumulative Impactfactor unterschieden sich nicht nur auf Ebene der einzelnen Organisationseinheiten, sondern der bereits angedeutet Kontrast zwischen den beiden Organisationsstrukturen spiegelte sich auch auf der Ebene der Leistungsfähigkeit der einzelnen Ärzte wieder. So publizierten die 55 Ober- und ChefärztInnen an den Lehrstühlen durchschnittlich 15,31 Publikationen pro Arzt im 5-Jahres-Zeitraum zwischen 2009 und 2013 (Range: 0-174 Publikationen/ 5 Jahre) ( $\bullet$ Abb. 4a). Im Gegensatz dazu veröffentlichte einer der 18 Ärzte einer untergeordneten Organisationsstruktur 5,78 Publikationen/5 Jahre (Range: 0-26 Publikationen/5 Jahre), was $37,8 \%$ der Publikationsanzahl der Ärzte an eigenständigen Kliniken entsprach. Der Unterschied war nicht signifikant $(p=0,066)$. Ein ähnliches Bild zeigte sich bei den kumulativen Impactfaktoren/Arzt in 5 Jahren ( $\bullet \mathbf{A b b}$. $\mathbf{4 b}$ ). Hier wurde bei den autonomen Kliniken pro Arzt ein durchschnittlicher kumulativer Impactfaktor von 28,4 Impactfaktorpunkten/5 Jahre von (Range: 0-327,7 Impactfaktorpunkte/5 Jahre) erzielt. Bei den anderen Fachgebieten untergeordneten Organisationsstrukturen wurden pro Arzt durchschnittlich 8,3 Impactfaktorpunkte/5 Jahre (Range: 0-55,05 Impactfaktorpunkte/5 Jahre) erreicht, was einem Anteil von $29,2 \%$ entsprach. Dieser Unterschied war nicht signifikant $(\mathrm{p}=0,051)$.

Die Auswertung der Impactfaktor-Punkte pro Publikation zeigt einen deutlichen Negativtrend insbesondere in Bezug auf die
Qualität der Veröffentlichungen der untergeordneten Organisationsstrukturen ( $\bullet$ Abb. 5). Während 2009 der Unterschied zwischen den Impactfaktorpunkten/Publikation noch nicht signifikant war, ist dieser Unterschied 2013 bereits hochsignifikant $(p<0,01)$ ausgeprägt, sodass die durchschnittlichen Impactfaktorpunkten/Publikation der untergeordneten Organisationsstrukturen mit 0,99 Impactfaktorpunkten/Publikation um 45,0\% niedriger lagen als die der eigenständigen Kliniken mit 1,80 Impactfaktorpunkten/Publikation.

Auch insgesamt lässt sich im 5-Jahresvergleich ein negativer Trend abzeichnen, sodass hier die durchschnittliche Publikation mit 2,04 Impactfaktorpunkten/Publikation (2009) um 17,6\% auf 1,68 Impactfaktorpunkten/Publikation (2013) sank. Bei den eigenständigen Kliniken ist dieser Trend weniger stark ausgeprägt. Hier sank im 5-Jahresvergleich der Wert von 2,05 Impactfaktorpunkten/Publikation (2009) um $12,2 \%$ auf 1,80 Impactfaktorpunkten/Publikation (2013). Im Gegensatz hierzu ist ein deutlicher Kontrast bei den untergeordneten Organisationsstrukturen zu verzeichnen. Hier sank im 5-Jahresvergleich der Wert von 1,92 Impactfaktorpunkten/Publikation (2009) um 48,4\% auf 0,99 Impactfaktorpunkten/Publikation (2013). Es zeigt sich mit einem Ausreißer von 2010 daher eine hochsignifikant zunehmende Schere in der Publikationsqualität zwischen autonomen Kliniken und anderen Fachgebieten untergeordneten Organisationsstrukturen.

Untersucht man die Verteilung der Publikationen nach Impactfaktoren zeigen sich einige interessante Details ( $\boldsymbol{0}$ Abb. 6). So ist die Hauptpublikationsleistung in den Jahren 2009-2013

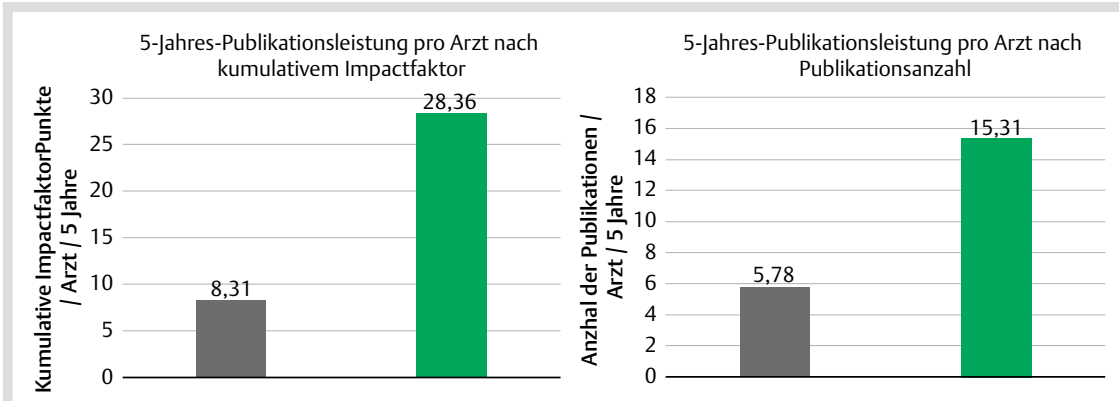

Abb. 4 5-jahres-Publikationsleistungpro Arzt (2009-2013). a Publikationsanzahl/Arzt/5 Jahre. b Kumulativer Impactfaktor/Arzt/5 Jahre Dunkelgrün: Eigenständige Kliniken, Grau: Untergeordnete Organisationsstrukturen, Stand: 10.06 .2014

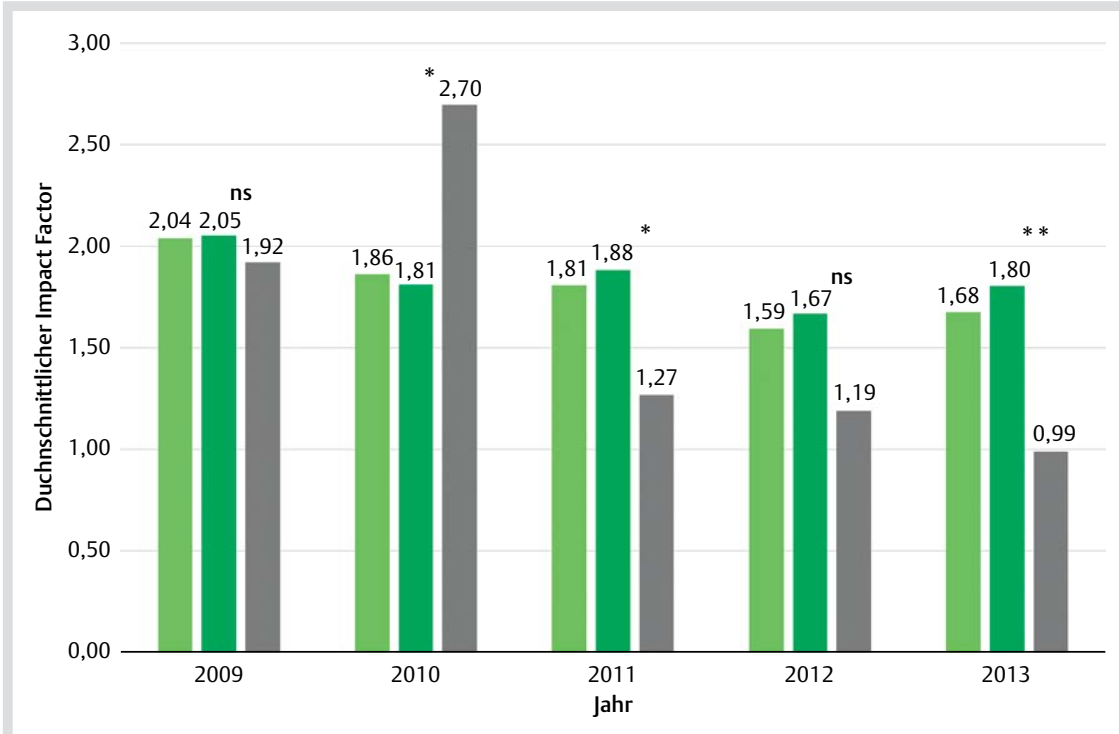

Abb. 5 Durchschnittliche Qualität der einzelnen Publikationen gemessen nach Impactfaktor pro Publikation. Hellgrün: Eigenständige Kliniken + untergeordneten Organisationsstrukturen, Dunkelgrün: Eigenständige Kliniken, Grau: Untergeordnete Organisationsstrukturen, Stand: 10.06.2014 


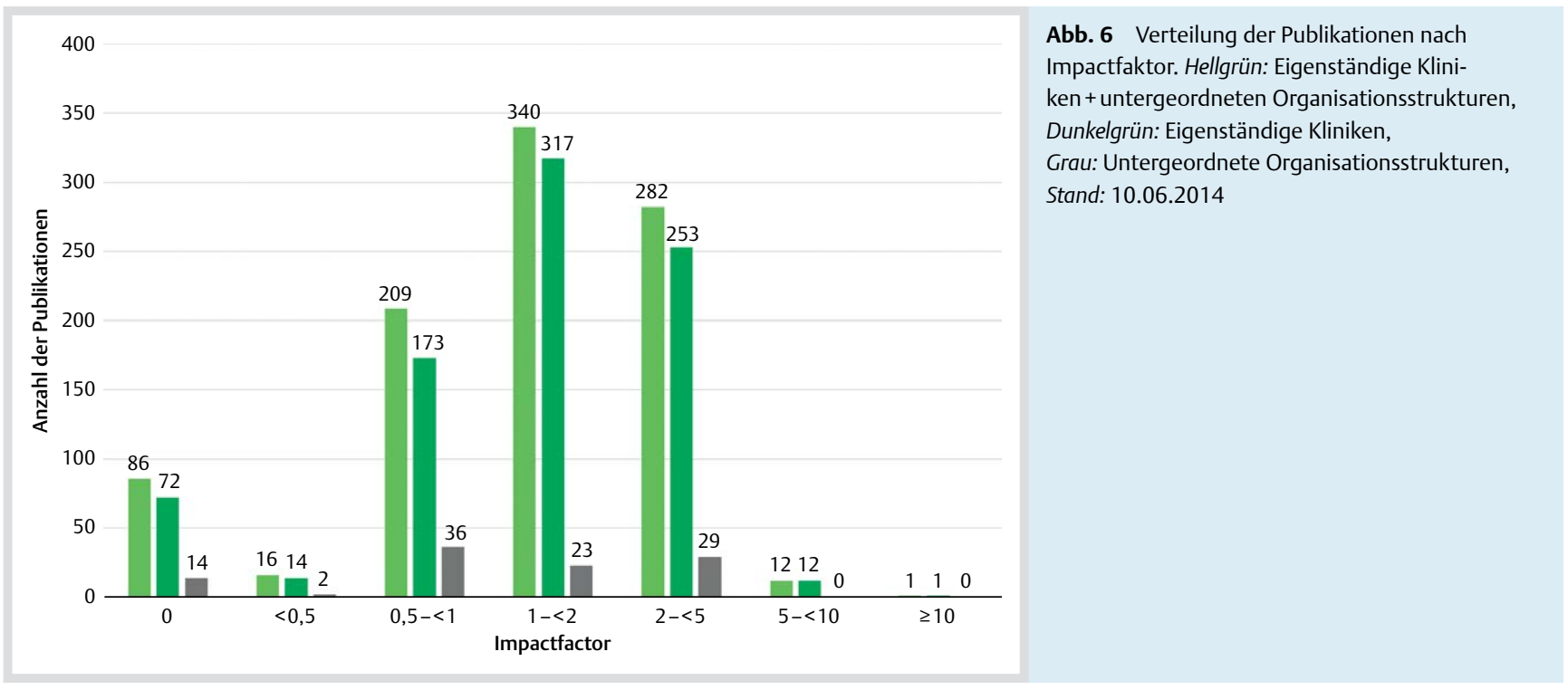

Tab. 2 Maximal- und Minimalwerte (Range) der graphisch dargestellten Werte.

\begin{tabular}{llll} 
& $\begin{array}{l}\text { Eigen- } \\
\text { ständige } \\
\text { Klinik }\end{array}$ & $\begin{array}{l}\text { Untergeordnete } \\
\text { Organisations- } \\
\text { strukturen }\end{array}$ & Gesamt \\
\hline IF/Publikation & $0-54,4$ & $0-4,1$ & $0-54,4$ \\
\hline Publikationsanzahl/Arzt/Jahr & $0-54$ & $0-6$ & $0-54$ \\
\hline Publikationsanzahl/Klinik/Jahr & $0-61$ & $0-17$ & $0-61$ \\
\hline Dargesteller Zeitram 2009-2013, Stand: $10.06,2014$ &
\end{tabular}

Dargestellter Zeitraum 2009-2013, Stand: 10.06.2014

zwischen den Impactfaktorenpunkten 0,5-5 angesiedelt. Das gilt sowohl für die eigenständigen Kliniken als auch für die untergeordneten Organisationsstrukturen. Mit insgesamt 86 Publikationen sind jedoch 9,1\% in Journals ohne Impactfaktor publiziert worden. Des Weiteren fällt auf, dass Spitzenpublikationen mit Impactfaktorpunkten über 5 lediglich durch die selbstständigen Kliniken veröffentlicht werden, sodass sich auch hier die soeben beschriebene Schere in der Publikationsleistung wiederspiegelt.

- Tab. 2 veranschaulicht die Bandbreite der oben dargestellten Ergebnisse. Auffallend ist hier, dass Top-Publikationen mit einem Impactfaktor von 54,4 Punkten lediglich an den autonomen Kliniken auftreten. Dennoch werden auch an einigen selbstständigen Lehrstühlen für Plastische Chirurgie Publikationen in Journals ohne Impactfaktor veröffentlicht. Bemerkenswert ist zudem, dass an eigenständigen Kliniken einzelne Ärzte mit maximal 54 Publikationen pro Jahr teils eine enorme Anzahl an Publikationen veröffentlichen, während dieser Trend bei den untergeordneten Organisationsstrukturen nicht in diesem extremen Sinne ausgeprägt ist. Entsprechend gehören auch mit 61 Publikationen pro Jahr und pro Klinik die wissenschaftlich produktivsten Kliniken zu den selbstständigen Kliniken. Dennoch ist auffallend, dass es auch unter den eigenständigen Kliniken einzelne Ärzte aber auch gesamte Kliniken gibt, die in einem Jahr kein einziges Paper veröffentlichen. Eine Beobachtung, die sich auch bei den untergeordneten Organisationsstrukturen zeigt.

\section{Journal-Analyse}

Die insgesamt 946 Publikationen sowohl der eigenständigen Kliniken als auch der untergeordneten Organisationsstrukturen im
5-Jahres-Zeitraum zwischen 2009-2013 verteilten sich auf 220 verschiedene Fachzeitschriften. 552 dieser Publikationen entfielen dabei auf die Top-10 Fachzeitschriften, was einem Anteil von $58,4 \%$ entspricht. Mehr als die Hälfte aller Publikationen wurden somit in diesen Top-10 veröffentlicht ( $\bullet$ Abb. 7). Unter den Top-10 wissenschaftlichen Fachzeitschriften finden sich 3 deutschsprachige und 7 englischsprachige Zeitschriften. Die ersten beiden Plätze werden von deutschsprachigen Zeitschriften belegt. Dennoch sind unter den Top-10 Fachzeitschriften mit insgesamt 331 bzw. einem Anteil von 60,0\% der Veröffentlichungen die englischsprachigen Zeitschriften im Vergleich zu den deutschsprachigen Zeitschriften mit 221 und einem Anteil von $40,0 \%$ der Publikationen führend, was die internationale Auslegung der deutschen universitären Forschung wiederspiegelt. Berechnet man den kumulativen Impactfaktor, der auf jedes der Top-10 Fachzeitschriften entfällt, ändert sich das Ranking zugunsten der englischsprachigen Fachzeitschriften ( $\bullet$ Abb. 8). Die ersten 3 Plätze werden hier von englischsprachigen Fachzeitschriften belegt. Auch wenn diese in der Publikationszahl hinter den deutschsprachigen Zeitschriften liegen, wird der Großteil der Impactfaktorpunkte in den englischsprachigen Journals generiert. In den gesamten Top-10 Fachzeitschriften werden 755,0 Impactfaktorpunkte und somit $44,2 \%$ der gesamten kumulativen Impactfaktorpunkte generiert. Davon entfällt mit 601,8 Impactfaktorpunkten mit 79,7\% der Großteil auf englischsprachige Titel. 153,2 Impactfaktorpunkte und somit 20,3\% entfallen auf deutschsprachige Fachzeitschriften.

In $\odot$ Abb. 9 wurde die Entwicklung der Top-10 Fachzeitschriften hinsichtlich ihrer jeweiligen Impactfaktor-Werte abgebildet. Über den Zeitraum von 5 Jahren konnten die gesamten Top-10 Zeitschriften ihren Impactfaktor von durchschnittlich 1,13 Impactfaktorpunkten (Range: 0-2,74) (2009) um +13,5\% (Range: -14 bis $+56 \%$ ) auf durchschnittlich 1,28 Impactfaktorpunkte (Range: 0-3,535) (2013) steigern ( $\bullet$ Abb. 8) was die zunehmende Qualität der beliebtesten plastisch-chirurgischen Fachzeitschriften untermauert. Die deutschsprachigen Fachzeitschriften steigerten ihren Impactfaktor um durchschnittlich 8,67\% (Range: -14 bis $+32 \%$ ), die englischsprachigen Titel um durchschnittlich $15,0 \%$ (Range: -8 bis $+56 \%$ ). 


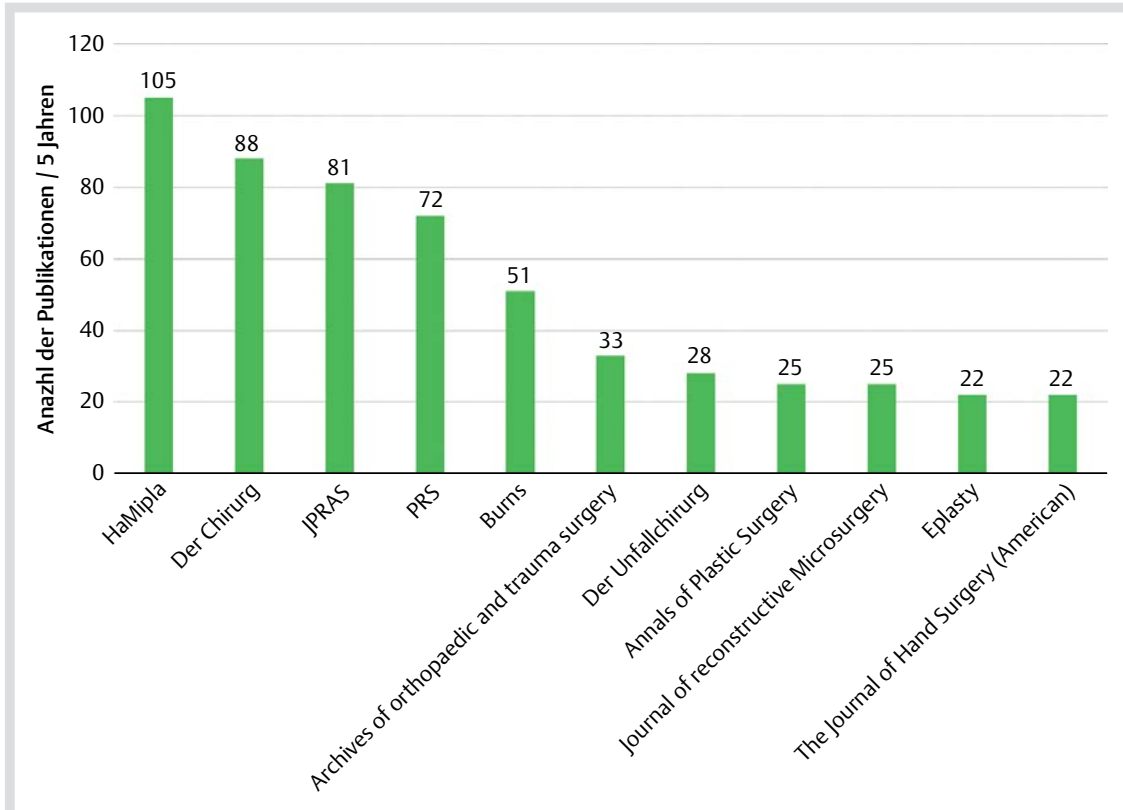

Abb. 7 Top-10 Fachzeitschriften der gesamten universitären Plastischen Chirurgie: Eigenständige Kliniken + untergeordnete Organisationsstrukturen, Anordnung nach Publikationszahl. Dargestellter Zeitraum 2009-2013, Stand: 10.06.2014

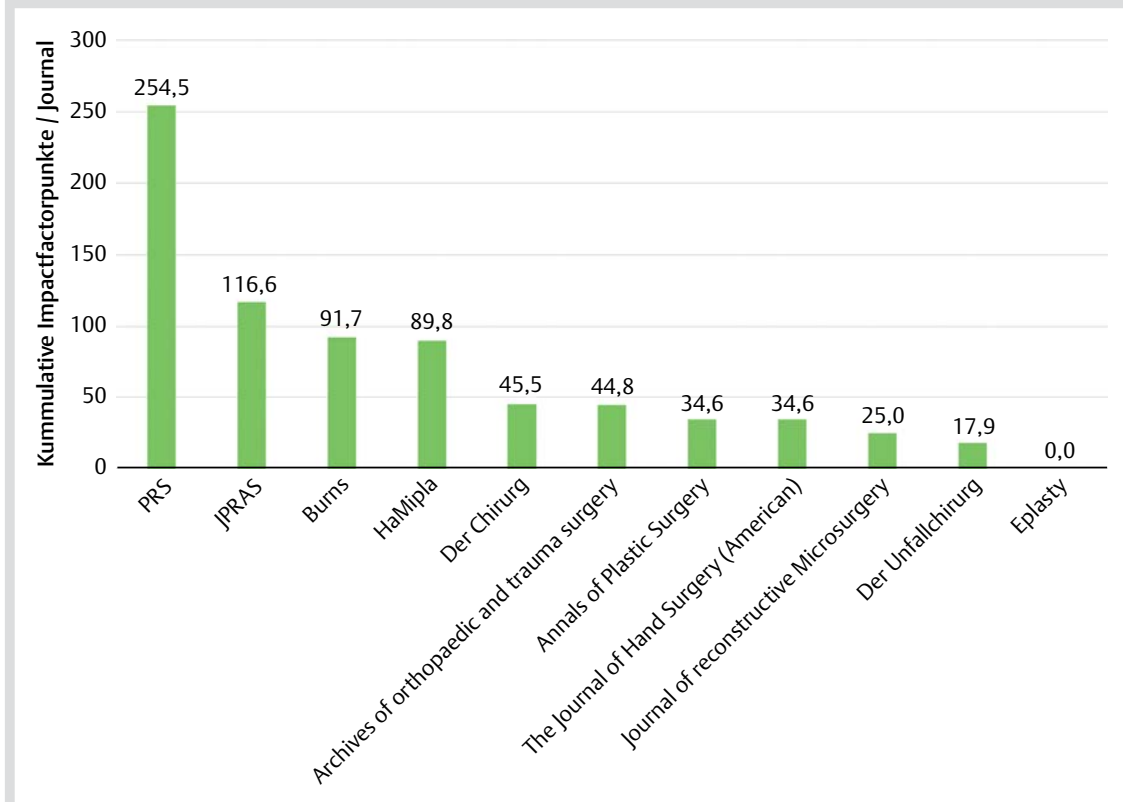

Abb. 8 Top-10 Fachzeitschriften der gesamten universitären Plastischen Chirurgie: Eigenständige Kliniken + untergeordnete Organisationsstrukturen, Anordnung nach kumulativem Impactfaktor. Dargestellter Zeitraum 2009-2013, Stand: 10.06 .2014

\section{Diskussion}

Der chirurgische Fachbereich der Plastischen Chirurgie hat sich in den vergangenen Jahren im klinischen wie auch im wissenschaftlichen Alltag zunehmend etabliert. Als gar nicht mehr so junges Fachgebiet - Eigener Facharzt seit 1993 - und „kleines“ Fach befindet es sich sowohl in Deutschland als auch in den USA in einem beständigen und dynamischen Wachstumsprozess mit dem Ziel, eine Starke Säule innerhalb der Familie aller chirurgischen Fachgebiete zu werden $[2,4,5]$. Dass eine zunehmende Autonomie des Faches vielseitige Vorteile bringt, ist unumstritten. Ein positiver Effekt einer zunehmenden Unabhängigkeit auf die wissenschaftliche Leistungsfähigkeit, ein zentraler Anspruch innerhalb der Universitätsmedizin, ist bereits ansatzweise beschrieben [2-4]. Ein messbarer Indikator für die wissenschaftliche Leistungsfähigkeit innerhalb der akademischen Plastischen Chirurgie stellt wiederum die Publikationsleistung einer jeden Klinik dar.
In dieser Arbeit konnten wir zeigen, dass I) eigenständige Kliniken mit Lehrstühlen für Plastische Chirurgie an deutschen Universitätsklinika nach wie vor unterrepräsentiert sind, dass II) ein signifikanter qualitativer und quantitativer Unterschied in der Publikationsleistung zwischen selbstständigen Kliniken und untergeordneten Organisationsstrukturen besteht, dass III) die allgemeine Publikationsleistung der akademischen Plastischen Chirurgie im 5-Jahres-Verlauf deutlich abnahm und dass IV) die Qualität der beliebtesten plastisch-chirurgischen Fachzeitschriften deutlich zunahm. In der Webseiten-Analyse stellte sich heraus, dass an lediglich 12 von 35 gewerteten Universitätsklinika Lehrstühle für Plastische Chirurgie etabliert sind ( $\bullet$ Abb. 1). An 76\% der Universitätsklinika ist die Plastische Chirurgie somit noch unterrepräsentiert. Dies betrifft unter anderem auch die personelle Ausstattung. So sind an den bereits existierenden Kliniken für Plastische Chirurgie mit durchschnittlich 3,6 OberärztInnen mehr als 3-mal so viele OberärztInnen beschäftigt als an 


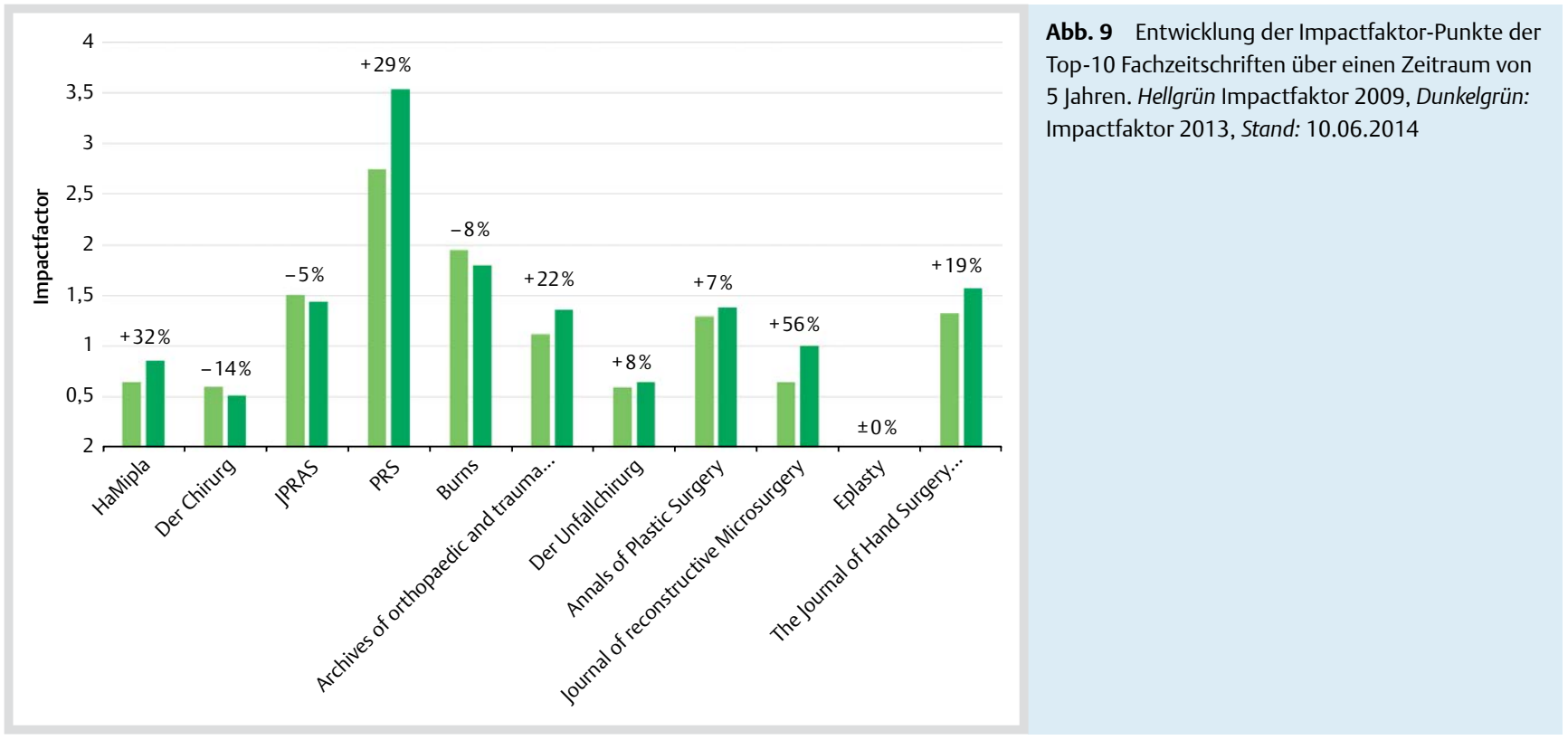

Unversitätskliniken, in denen die Plastische Chirurgie im Rahmen von anderen Fachgebieten untergeordneten Organisationsstrukturen geführt wird. Dieser Ist-Zustand der Universitätsklinik-Landschaft spiegelt einen deutlichen Nachholbedarf im Sinne einer aktuell noch mangelnden Autonomie der Plastischen und Ästhetischen Chirurgie dar. Ausreichende personelle Ressourcen zusammen mit finanzieller, akademischer, administrativer Eigenständigkeit sowie der Beteiligung an leistungsorientierten Mitteln bilden jedoch eine Grundlage für ein erfolgreiches und gerechtfertigtes Wachstum des Faches [4]. Sicherlich ist hier zeitnah die Etablierung weiterer Lehrstühle für Plastische Chirurgie sowohl für eine professionelle interdisziplinäre Patientenversorgung als auch für die akademische und klinische Emanzipation des Fachgebietes unerlässlich.

Die Pubmed-Analyse der Publikationsleistung im 5-Jahres-Zeitraum zwischen 2009 und 2013 zeigt einen signifikanten Unterschied abhängig von der jeweiligen Organisationsstruktur (○ Abb. 2-5, $\odot$ Tab. 2). So entfiel mit 89,0\% (○ Abb. 2) der Großteil der Publikationsanzahl, sowie mit 91,2\% ein noch größerer Anteil der kumulativen Impactfaktorpunkte auf die selbstständigen Lehrstühle ( $\triangle$ Abb. 3). Dort werden auch die meisten Publikationen pro Arzt (54 Publikationen/Jahr) und pro Klinik (61 Publikationen/Jahr) generiert ( $\bullet$ Tab. 2). Im Gegensatz dazu ist die Publikationsleistung an untergeordneten Organisationsstrukturen vergleichbar gering. Nur 11,0\% der Publikationsanzahl und lediglich $8,8 \%$ der kumulativen Impactfaktorpunkte entfielen auf die untergeordneten Organisationsstrukturen (๑ Abb. 2, 3). Dieser Kontrast war jedoch nicht alleine auf die bessere personelle Ausstattung der Lehrstühle zurückzuführen, sondern zeichnete sich auch bei der wissenschaftlichen Leistungsfähigkeit der jeweils einzelnen Ärzte ab ( $\bullet$ Abb. 4a, b). Obgleich der Unterschied nicht signifikant war, führten auch hier die selbstständigen Lehrstühle. So konnte in 5 Jahren ein durchschnittlicher Arzt an untergeordneten Einheiten lediglich 37,8\% der Publikationsanzahl sowie nur 29,2\% des kumulativen Impactfaktors der Kollegen an selbstständigen Kliniken erreichen. Auch Top-Publikationen in hochrangigen Fachzeitschriften mit einem Impactfaktor $>5$ als Zeichen einer hochwertigen und international wettbewerbsfähigen Forschung sind ausschließlich an eigenständigen Kliniken für Plastische Chirurgie zu finden und werden von den untergeordneten Organisationsstrukturen nicht erreicht $(\triangle$ Abb. 5, 6). Dies ist ein Hinweis dafür, dass hochqualifizierte Forschung nur an Organisationsstrukturen möglich ist, die entsprechend einem unabhängigem Fachgebiet angemessen ist. Eine deutliche Schere in der wissenschaftlichen Leistungsfähigkeit ist daher offensichtlich. Dieser Effekt ist insbesondere im 5-Jahres-Verlauf auffällig ( $\bullet$ Abb. 5). Während der durchschnittliche Impactfaktor an eigenständigen Kliniken um $12,2 \%$ sank, brach der durchschnittliche Impactfaktor an untergeordneten Organisationsstrukturen um 48,4\% gravierend ein, was auf einen Einbruch in der Forschungsqualität deutet. Obgleich die eigenständigen Kliniken im Vergleich zu den untergeordneten Organisationsstrukturen über eine deutlich potentere Publikationsleistung verfügen, ist aber auch hier ein deutlicher Negativtrend ersichtlich. Insgesamt waren über den Analysezeitraum von 5 Jahren sowohl die Publikationszahl (-13,4\%) als auch die kumulativen Impactfaktorpunkte (-28,9\%) sowie die Impactfaktorpunkte pro Publikation $(-17,6 \%)$ rückläufig ( $\bullet$ Abb. 2-5). Dies spiegelt einen deutlichen Rückgang der Gesamtleistungsfähigkeit der universitären Plastischen Chirurgie an deutschen Universitätsklinika dar. Obwohl hier weiterführende Untersuchungen nötig sind, um die Gründe diesbezüglich weiter zu differenzieren, ist bereits jetzt klar, dass dringend stärkere Anreize zur Forschungsförderung sowie bessere berufliche Perspektiven insbesondere für den wissenschaftlichen Nachwuchs nötig sind. Mangelnde Freistellung, ausgedehnte bürokratische Hürden, mangelnde Finanzierungsmöglichkeiten oder zu hohe Anforderungen beim Habilitationsverfahren könnten hier weitere Gründe für ein rückläufiges Forschungsengagement sein. In der Journal-Analyse zeigte sich, dass mit 58,4\% über die Hälfte der Publikationen auf lediglich 10 von insgesamt 220 unterschiedlichen Fachzeitschriften entfielen ( $\bullet$ Abb. 7). Diese Top-10 der beliebtesten Fachzeitschriften wurde bezüglich der Publikationszahl von 2 deutschsprachigen Titeln angeführt ( $\bullet$ Abb. 7). Im Gegensatz dazu wurde jedoch der höchste kumulative Impactfaktor eben nicht bei diesen Titeln erzielt, sondern von den englischsprachigen Fachzeitschriften generiert ( $\bullet$ Abb. 8), was mit der entsprechend höheren Impactfaktor-Bewertung der 
englischsprachigen Titel zu erklären ist. Diese Analyse zeigt, dass die deutsche akademische Plastische Chirurgie international orientiert ist und dort deutlich sichtbar repräsentiert ist. Innerhalb der Top-10 werden bezüglich der Publikationsanzahl 60\% in englischsprachigen Fachzeitschriften veröffentlicht. Der kumulative Impactfaktor spiegelt ein noch deutlicheres Bild wieder. So werden innerhalb der Top-10 der Fachzeitschriften 79,9\% des kumulativen Impactfaktors in den englischsprachigen Zeitschriften generiert. Obgleich die deutschsprachigen Fachzeitschriften hinsichtlich des Impactfaktors den englischsprachigen Titeln unterlegen sind, ist im 5-Jahresvergleich auch hier ein Positivtrend ersichtlich ( $\bullet$ Abb. 9). So konnten zwischen 20092013 die deutschsprachigen Fachzeitschriften ihren Impactfaktor um durchschnittlich 8,67\% steigern. Bei den englischsprachigen Titeln war dieser Positivtrend noch ausgeprägter mit einem durchschnittlichen Zuwachs von 15,0\%. Insgesamt konnte innerhalb der Top-10 damit der durchschnittliche Impactfaktor um $13,5 \%$ von 1,13 auf 1,28 Impactfaktorpunkte gesteigert werden, was einen sichtbaren Anstieg der Qualität und Anerkennung dieser Zeitschriften und indirekt damit auch der Plastisch-Chirurgischen Forschung innerhalb der internationalen Forschungsgemeinschaft darstellt.

\section{Schlussfolgerung}

Zusammenfassend zeigt diese Arbeit, dass die Art der Organisationsstruktur neben anderen Einflussgrößen sicherlich einen deutlichen Einfluss auf die publizierte Leistungsfähigkeit der akademischen Plastischen Chirurgie in Deutschland hat. So fördert eine bessere Ressourcen-Ausstattung im Sinne autonomer Lehrstühle die Publikationszahl sowohl pro Arzt als auch pro Klinik sowie die Höhe des kumulativen Impactfaktors und trägt damit erheblich zum wissenschaftlichen Ergebnis der Chirurgie an einer Universitätsklinik bei. Desweitern sind Top-Publikationen mit hohen Impactfaktoren als Zeichen einer professionellen und international wettbewerbsfähigen Forschung nur an eigenständigen Kliniken zu finden. Der Kontrast zwischen selbstständigen Kliniken und anderen Fachrichtungen untergeordneten Organisationsstrukturen hat sich in den vergangenen 5 Jahren zunehmend verschärft und droht in einer wissenschaftlichen 2-Klassen-Gesellschaft innerhalb der akademischen Plastischen Chirurgie zu münden. Diesen Trend zusammen mit einer allgemeinen Abnahme der Leistungsfähigkeit der deutschen akademischen Plastischen Chirurgie gilt es kritisch zu hinterfragen und konsequent entgegen zu steuern. Eine regelmäßige, möglichst jährliche Dokumentation der Publikationsleistung der akademischen Plastischen Chirurgie erscheint uns zur zeitgemäßen Optimierung der Organisationsstrukturen an Universitätsklinik besonders wichtig. So könnte bspw. durch die Einrichtung eines Registers zur Erfassung der Publikations- und Mitarbeiterzahlen einer jeden universitären Organisationseinheit unkompliziert und verlässlich eine nationale Statistik bezüglich der aktuellen Publikationsleistung generiert werden. Zugleich bieten sich durch ein Register Möglichkeiten eines anonymen Benchmarking für jede einzelne Organisationseinheit. Insgesamt könnte ein solcher gemeinschaftlicher Vorstoß, auch von anderen Fachgebieten wahrgenommen werden und bei der Argumentation zur Einrichtung neuer Lehrstühle hilfreich sein.

\section{Anmerkung \\ $\nabla$}

Die Erstautorenschaft wird zwischen den Autoren Cornelius D. Schubert und Sebastian Leitsch geteilt.

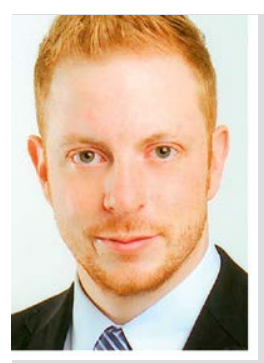

Cornelius D. Schubert

Studium der Humanmedizin an der Universität Regensburg mit Auslandsaufenthalten u. A. in Boston (Brigham and Women's Hospital, Harvard Medical School) und Baltimore (Johns Hopkins Hospital, Johns Hopkins School of Medicine). Approbation 2012. Experimentelle Doktorarbeit an der Klinik für Plastische Chirurgie \& Schwerbrandver-

letzte, Handchirurgie-Zentrum des BG Universitätsklinikum Bergmannsheil GmbH (Ruhr-Universität Bochum).

Promotion 2013. Seit 2013: Assistenzarzt in Weiterbildung zum Facharzt für Plastische und Ästhetische Chirurgie am Klinikum der Ludwig-Maximilians Universität München (Chefarzt: Univ.-Prof. Dr. med. Riccardo E. Giunta).

Interessenkonflikt: Nein

\section{Literatur}

1 Giunta RE. Ursula Schmidt-Tintemann-pioneer of plastic surgery in Germany. Handchir Mikrochir Plast Chir 2008; 40: 408-410

2 Giunta RE, Machens HG. Science and research in academic plastic surgery in Germany. Handchir Mikrochir Plast Chir 2009; 41: 359-363

3 Koeppel TA, Eckstein HH, Florek HJ et al. Akademische Gefäßchirurgie in Deutschland: dringender Bedarf für ein strukturiertes Ausbildungsprogramm. Gefässchirurgie 2008; 13: 265-272

4 Rohrich RJ. Mandating Departments of Plastic Surgery: the future of plastic surgery is now. Plast Reconstr Surg 2008; 121: 1499-1502

5 Stokes $M$. Case Western Reserve medical school establishes department of plastic surgery. Plastic Surgery News 2007; 18

6 [Anonym]. Verband der Universitätsklinika Deutschlands e.V., - VUD. In: Verband der Universitätsklinika Deutschlands e.V., - VUD 2013 